\section{Barreras de protección utilizadas por odontólogos en México, durante la pandemia por COVID-19}

\author{
Protection barriers used by dentists in \\ Mexico during the COVID-19 pandemic
}

\begin{abstract}
Resumen
Objetivo. Comparar el tipo de barreras de protección utilizadas en consultorios dentales de México durante la pandemia. Métodos. Se realizó un estudio transversal en 250 odontólogos que laboran en instituciones, consultorios o clínicas particulares de cinco estados de la república mexicana; la información se recolectó en un formulario de Google que incluyó: consentimiento informado, ficha de identificación, uso de barreras de protección y cambio de las mismas. Se realizó un análisis descriptivo de los datos. Resultados. Fueron 250 participantes, 50 de cada estado, la media de la edad fue 37,10 ańos (DE 11,98), predominó el sexo femenino, la condición de soltero, y el nivel de estudios de licenciatura. La barrera más utilizada fue gorro quirúrgico y/o desechable, señalaron atender pacientes con protección ocular y careta más del $80 \%$; el uso de la mascarilla KN95/N95 fue mayor que la de triple capa, sólo el 3\% respondió no usar guantes, fue elevado el empleo de bata desechable, 31 participantes aún no han atendido pacientes. La mayoría cambian gorro, careta, mascarilla y bata con cada paciente, refirieron incrementar el uso de barreras durante la pandemia. Hubo significancia estadística entre grupos de edad, estado civil y nivel de estudios respecto a la entidad. Conclusiones. El uso de las barreras de protección fue elevado, el equipo más empleado fue la protección ocular, careta, mascarillas, guantes, bata y campos desechables; sin embargo, es necesario se incremente el cambio de barreras después de cada paciente.
\end{abstract}

Palabras clave: COVID-19; Equipo de protección personal; Consultorios odontológicos (fuente: DeCS BIREME).

\begin{abstract}
Objective. Compare the type of protective barriers used in dental clinics in Mexico during the pandemic. Methods. A cross-sectional study was performed with 250 dentists who work in institutions, clinics, or private offices in five states of the Mexican Republic; the information was collected in a Google form that included: informed consent, identification card, use of protection barriers and protocol to use them. A descriptive analysis of the data was prepared. Results. There were 250 participants, 50 from each state, the mean age was 37.10 (SD 11.98), predominating, females, single status and having a bachelor's degree. The most used barrier was a surgical and/or disposable cap, more than
\end{abstract}

\section{Artículo Original}

Christian Starlight Franco-Trejo 1,a, Rosa Alicia

García-Jau 2,a , Aurora Lucero-Reyes ${ }^{3, b}$, Guadalupe

Rosalia Capetillo-Hernández ${ }^{4, a}$, Rosendo Gerardo

Carrasco-Gutiérrez ${ }^{5, c}$, Ana Karenn González Álvarez ${ }^{6, b}$

${ }^{1}$ Universidad Autónoma de Zacatecas, Maestría en Ciencias de la Salud, Unidad Académica de Medicina Humana, Zacatecas, México.

2 Universidad Autónoma de Sinaloa, Facultad de Odontología, Sinaloa, México.

${ }^{3}$ Universidad Autónoma de Tlaxcala, Facultad de Odontología, Tlaxcala, México.

${ }^{4}$ Universidad Veracruzana, Facultad de Odontología, Veracruz, México.

${ }^{5}$ Benemérita Universidad Autónoma de Puebla, Facultad de Odontología, Puebla, México.

${ }^{6}$ Universidad Autónoma de Zacatecas, Unidad Académica de Odontología, Zacatecas, México.

a Doctora en Ciencias.

${ }^{\mathrm{b}}$ Maestra en Ciencias

${ }^{\mathrm{c}}$ Maestro en Ciencias.

\section{Correspondencia:}

Christian Starlight Franco Trejo: fatc007964@uaz.edu.mx Campus Universitario Siglo XXI, edificio L1, segundo piso, carretera Zacatecas-Guadalajara, kilómetro 6, Ejido La Escondida, Zacatecas, Zacatecas, C.P. 98618. ORCID: 0000-0002-4250-5483

\section{Coautores:}

Rosa Alicia García-Jau: rossygaja@hotmail.com ORCID: 0000-0002-7221-3764

Aurora Lucero-Reyes: auroraluceroreyes@gmail.com ORCID: 0000-0002-9841-779X

Guadalupe Rosalia Capetillo-Hernández: gcapetilloh@ hotmail.com

ORCID: 0000-0002-2033-4660

Rosendo Gerardo Carrasco-Gutiérrez: rosendocarrascogutierrez@gmail.com

ORCID: 0000-0001-7680-3094

Ana Karenn González Álvarez: dra.ana.karenn.gonzalez@ uaz.edu.mx

ORCID: 0000-0001-9015-1141

\section{Editora:}

Antonieta Pérez-Flores

Universidad de Concepción, Chile

Conflicto de intereses: Los autores declaran no tener conflictos de interés.

Fuente de financiamiento: Autofinanciado.

Recibido: 20/10/21

Aceptado: 09/12/21

Publicado: 21/01/22 
$80 \%$ indicated servicing patients using eye protection and a mask; the use of the KN95 / N95 mask was greater than the triple layer, only 3\% responded not to use gloves, the use of disposable gowns was high, and 31 participants have not seen patients yet. The majority $(48.5 \%)$ change hat, face shield, mask, and gown with each patient. They referred to had increased the use of barriers during the pandemic. There was statistical significance between age groups, marital status and educational level with respect to the entity. Conclusions. The use of protective barriers was elevated, the most used equipment was eye protection, face shield, masks, gloves, gown, and disposable fields; however, it is necessary to increase the change of barriers after each patient.

Keywords: COVID-19; Personal protective equipment; Dental clinic (source: $\mathrm{MeSH}$ NLM).

\section{Introducción}

Dentro del trabajo asistencial, el odontólogo juega un papel importante en la trasmisión de infecciones cruzadas, está expuesto ante una variedad de microorganismos que ocasionan enfermedades, por el uso de instrumentos cortantes y el contacto con fluidos orgánicos contaminados, además de la transmisión aérea por microgotas generadas al hablar, toser o por el uso de turbinas, todo ello conlleva un riesgo de transmisión de infecciones tanto al personal como al paciente ${ }^{1,2}$. La Organización Mundial de la Salud (OMS) ha alertado sobre la falta de conciencia de trabajadores y estudiantes de la salud, y del público en general, sobre procedimientos inadecuados de prevención y control que pueden provocar brotes basados en infecciones nosocomiales ${ }^{3}$. La exposición durante la consulta dental a microorganismos que producen tuberculosis, hepatitis, VIH/SIDA, SARS, ébola, y recientemente COVID-19, con el uso correcto de barreras de protección se puede disminuir el riesgo de contagio para el odontólogo y los pacientes ${ }^{4}$. Dado que la transmisión del SARS-CoV-2 se da principalmente por contacto directo e indirecto, es imprescindible que el dentista se proteja ante el contagio, con el uso adecuado del equipo de protección personal (EPP), que incluye gafas protectoras, mascarillas, guantes, gorros, protectores faciales y ropa protectora; aunque éstos reducen el riesgo de transmisión no pueden proporcionar una barrera a prueba de ellas ${ }^{5}$.

Algunas investigaciones publicadas sobre el uso de las barreras de protección en odontología, fueron Camargo et al. ${ }^{6}$ donde encontró que el $91,35 \%$ utilizó la bata indicada, el $89,42 \%$ gorro, uso adecuado de tapabocas con $71,15 \%$ y $97,12 \%$ guantes con cada paciente; Díaz 7, en la Universidad de Chile, identificó 63\% de barreras de protección utilizadas, entre ellas: guantes, mascarilla, gorro, lentes protectores, pechera. En tanto que $\mathrm{Paz}^{8}$ calificó la bioseguridad personal con $98,8 \%$, al igual que Assiri et al. ${ }^{9}$ con un alto cumplimiento en el uso de las barreras de protección a excepción de las gafas siendo utilizadas solo por el 22\%; mientras que, para Serrano et al. ${ }^{10}$ encontró nivel adecuado en $84 \%$ el uso de barreras. Pastor ${ }^{11}$ descubrió un nivel de conocimientos bueno sólo en el $13,7 \%$ sobre el uso de EPP, además, que fue deficiente entre más años de ejercicio profesional se tenga; Vazquez et al. ${ }^{12}$ en el área de posgrado se apegó en $34,4 \%$ a los protocolos de salud ocupacional, con una adherencia en la protección personal de 41,1\%. En Colombia durante la pandemia por COVID-19, Barbosa et al. ${ }^{13}$ determinó que $89,65 \%$ utilizó N95 o similares y mascarilla quirúrgica el $43,97 \%$, bata desechable antifluido estéril o no el $100 \%$, guantes desechables estériles el $22,89 \%$.

Con la aparición de la pandemia ocasionada por el virus SARS-CoV-2, en México a mediados del mes de marzo de 2020 se publicó en el Diario Oficial de la Federación (DOF) el acuerdo que señaló "...se suspenden las clases en las escuelas de educación preescolar, primaria, secundaria, normal y demás para la formación de maestros educación básica del sistema educativo nacional, así como aquellas de los tipos medio superior y superior dependientes de la secretaria de educación pública" ${ }^{14}$ como medida preventiva se cerraron escuelas además de algunos comercios para evitar la propagación de los contagios y el número de casos en el país, situación que afectó la economía familiar y social, sin excluir a los profesionales de la salud bucal, en caso de atender urgencias odontológicas debían incrementar los EPP durante la atención ${ }^{14}$.

En cuanto al personal odontológico que labora dentro de instituciones tanto educativas como de salud (derechohabiencia o gubernamentales), la mayoría se aisló durante las primeras semanas de la contingencia; posteriormente se incorporó de forma escalonada, pero sin atención a pacientes, a menos que fuera una urgencia. Esta situación no se presentó en por un largo periodo en clínicas y consultorios particulares, ya que, por las condiciones económicas y sociales, así como los compromisos con pacientes se tuvieron que tomar ciertas precauciones para continuar con sus actividades. Según Cázares et al. ${ }^{15}$ el impacto económico, derivado de la pandemia afectó al gremio odontológico en lo público y privado, principalmente por la adquisición de los EPP.

México, conocido de manera oficial como Estados Unidos Mexicanos, está conformado por 32 entidades federativas ${ }^{16}$, para esta investigación fueron seleccionados cinco estados por la vinculación académica de colegas que apoyaron con la difusión del formulario, localizados en el centro, centro-norte y este de la república mexicana. Por lo anterior, se motivó a realizar la presente investigación con el objetivo de comparar el tipo de barreras de protección utilizadas dentro de los consultorios dentales de cinco estados de México durante la pandemia. 


\section{Métodos}

Se realizó un estudio transversal en cirujanos dentistas que laboran en cinco Estados de la República Mexicana: Zacatecas, Sinaloa, Veracruz, Tlaxcala y Puebla, mismos que fueron elegidos por el vínculo con colegas que participaron para difundir el instrumento de recolección de información; se utilizó un muestreo no probabilístico por cuotas, con un tamaño de la muestra de 250 , donde se incluyeron 50 participantes por cada entidad durante el mes de marzo de 2021. Se consideraron odontólogos con práctica clínica mínima de un año, que laboraban a nivel institucional (de derechohabiencia, gubernamentales o educativas), en consultorios o clínicas particulares.

La recolección se realizó mediante un formulario en Google, dividido en tres apartados: consentimiento informado, ficha de identificación, items sobre uso de barreras de protección y cambio de las mismas. Dentro del cuestionario se explicó el uso y la privacidad de la información recabada, tomando en consideración el resguardo y cuidado de los datos sensibles recabados previo consentimiento informado donde se especificó el manejo de los mismos, con base en el Reglamento de la Ley General de Salud en materia de investigación para la salud ${ }^{17}$, que señalan que el estudio no es de riesgo, previo a la aplicación se obtuvo la aprobación y registro del proyecto dentro de las instancias universitarias con número de registro UAZ-2020-38288, avalado por el Comité de Investigación de la Unidad Académica de Odontología (UAO), de la Universidad Autónoma de Zacatecas (UAZ) así como de la Coordinación de Investigación y Posgrado de la misma universidad.

Las variables incluidas fueron edad, estado de residencia, estado civil, tiempo laborando además de nivel de estudios, mientras que los ítems sobre las barreras de protección: gorro quirúrgico, gorro desechable, protección ocular, careta, mascarilla quirúrgica, mascarilla de alta filtración, respiradores, cubrecalzado, guantes, doble guante, bata desechable, traje aislante, babero, campos desechables, plástico anti-adherente, funda desechable; éstos se obtuvieron de lo establecido a mediados del 2020 por parte de la subdirección de prevención y protección sobre la semaforización para retomar la práctica odontológica en México ${ }^{18}$, además de las recomendaciones para la práctica odontológica ${ }^{19}$ Respecto al cambio de barreras, se incluyeron dos preguntas de elaboración propia sobre la frecuencia y si se incrementaron durante la pandemia, siendo de elaboración propia y dicotómica.

Con la información recabada se generó una base de datos en el paquete estadístico SPSS v. 22 para el procesamiento, análisis y presentación de la información mediante estadística descriptiva. Se aplicó la prueba chi-cuadrada con la finalidad de encontrar diferencias entre los participantes de los diferentes estados según sexo, edad, estado civil y nivel de estudios. Se trabajó con un nivel de significancia del 5\%.

\section{Resultados}

Se obtuvo una muestra de 250 odontólogos, 50 de cada estado: 34,36 DE 9,46 en Zacatecas, 37,58 DE 10,54 Sinaloa, 44,52 DE 13,44 Veracruz, 31,90 DE 11,24 Tlaxcala y 37,00 DE 11,08 años de práctica en Puebla. El grupo de edad más frecuente fue de 20 a 29 años en Zacatecas, Tlaxcala y Puebla, mientras que en Sinaloa fue de 40-49 y en Veracruz fue de 30 a 39 años. Predominó el sexo femenino en los cinco estados, así como el estado civil de soltero en Zacatecas, Tlaxcala y Puebla; en tanto que en Sinaloa y Veracruz la mayoría respondió estar casado. Respecto al nivel de estudios lo más frecuente fue la licenciatura, solamente en Veracruz predominó el grado de doctor con 32\%. Hubo diferencia entre los estados respecto a la proporción de participantes por edad, estado civil y nivel de estudios (Tabla 1).

Dentro de los resultados obtenidos, en el total de los odontólogos se encontró que la mayoría de los participantes usaron gorro quirúrgico y/o desechable, más del $80 \%$ señalaron atender pacientes con protección ocular y careta; fue más elevado el uso de la mascarilla KN95/ N95 que la de triple capa, sólo el 3\% respondió no usar guantes mientras el 39,1\% lo hacía con doble guante. Fue elevado el empleo de bata desechable, aunque el $51,1 \%$ utilizó el traje aislante; el uso del babero y de campos tuvieron respuestas de $76,4 \%$ y $85,5 \%$, respectivamente (Tabla 2). Cabe señalar que 31 de los participantes aún no han atendido pacientes.

Al comparar las barreras usadas en diferentes entidades se encontró que el uso del gorro desechable y quirúrgico oscilo de $64 \%$ hasta el $76 \%$, la protección ocular fue elevada en todos los Estados, sólo en Zacatecas tres personas mencionaron no usarla (Tabla 3). En Sinaloa se presentó el mayor porcentaje con un $88 \%$ con mayor uso de las mascarillas KN95/N95 (Tabla 4) teniendo un porcentaje similar en Puebla con 84\%; 70\% de los zacatecanos indicó el uso de mascarilla triple capa, siendo esta mayor que en los otros lugares. Los respiradores son más usados en Veracruz (Tabla 5) en un 20\%. Siete odontólogos que residen en Zacatecas, Veracruz y Tlaxcala respondieron no usar guantes, lo que corresponde a el valor mayor de $8,0 \%$ en Tlaxcala (Tabla 6), el uso del doble guante fue más común en Tlaxcala con 54,0\%. La bata desechable con mayor porcentaje estuvo en $\mathrm{Za}$ catecas con $78 \%$ mientras el traje aislante fue $66 \%$ en Sinaloa. Los profesionales que laboran en Puebla (Tabla 7) coinciden sobre el uso frecuente de protección ocular, guantes y campos desechables.

Se preguntó sobre la frecuencia que se cambian materiales de protección como gorro, careta, mascarilla y bata, a lo que respondieron que con cada pacientes $48,5 \%$ (121), al término de la jornada el 49,4\% (124), cuando se dañan $1,3 \%$ (3) y que no los usan $0.9 \%$ (2); al comparar las respuestas según los estados se encontró que el $58 \%$ de los médicos tlaxcaltecas las cambian con cada paciente mientras Zacatecas y Sinaloa tuvieron el mismo porcentaje (50\%), la opción de al término de la jornada fue más elevado en Puebla con 63,6\%, y en 
Franco-Trejo et al.

Tabla 1. Características de los odontólogos por estados

\begin{tabular}{|c|c|c|c|c|c|c|c|c|c|c|c|}
\hline \multirow{3}{*}{ Variable } & \multicolumn{10}{|c|}{ Estado } & \multirow{3}{*}{$p$} \\
\hline & \multicolumn{2}{|c|}{ Zacatecas } & \multicolumn{2}{|c|}{ Sinaloa } & \multicolumn{2}{|c|}{ Veracruz } & \multicolumn{2}{|r|}{ Tlaxcala } & \multicolumn{2}{|c|}{ Puebla } & \\
\hline & $\mathbf{n}$ & $\%$ & $\mathbf{n}$ & $\%$ & $\mathbf{n}$ & $\%$ & $\mathbf{n}$ & $\%$ & n & $\%$ & \\
\hline Sexo & & & & & & & & & & & 0216 \\
\hline Masculino & 15 & 30,0 & 13 & 26,0 & 19 & 38,0 & 17 & 34,0 & 8 & 15,2 & 0,216 \\
\hline Femenino & 35 & 70,0 & 37 & 74,0 & 31 & 62,0 & 33 & 66,0 & 42 & 84,8 & \\
\hline \multicolumn{12}{|l|}{ Edad } \\
\hline $20-29$ & 19 & 38,0 & 14 & 28,0 & 8 & 16,0 & 31 & 62,0 & 18 & 36,4 & \\
\hline $30-39$ & 15 & 30,0 & 14 & 28,0 & 13 & 26,0 & 5 & 10,0 & 14 & 27,3 & $<0,001^{*}$ \\
\hline $40-49$ & 11 & 22,0 & 17 & 34,0 & 10 & 20,0 & 10 & 20,0 & 10 & 21,2 & \\
\hline 50 y más & 5 & 10,0 & 5 & 10,0 & 19 & 38,0 & 4 & 8,0 & 5 & 15,2 & \\
\hline \multicolumn{12}{|l|}{ Estado civil } \\
\hline Soltero & 26 & 52,0 & 18 & 36,0 & 20 & 40,0 & 34 & 68,0 & 27 & 54,5 & \\
\hline Casado & 19 & 38,0 & 27 & 54,0 & 26 & 52,0 & 8 & 16,0 & 15 & 30,3 & $0,007^{*}$ \\
\hline Otro & 5 & 10,0 & 5 & 10,0 & 4 & 8,0 & 8 & 16,0 & 8 & 15,2 & \\
\hline \multicolumn{12}{|c|}{ Nivel de estudios } \\
\hline Licenciatura & 28 & 56,0 & 26 & 52,0 & 15 & 30,0 & 41 & 82,0 & 18 & 36,4 & \\
\hline Especialidad & 13 & 26,0 & 5 & 10,0 & 14 & 28,0 & 5 & 10,0 & 11 & 21,2 & $<0,001^{*}$ \\
\hline Maestría & 6 & 12,0 & 12 & 24,0 & 5 & 10,0 & 4 & 8,0 & 17 & 33,3 & \\
\hline Doctorado & 3 & 6,0 & 7 & 14,0 & 16 & 32,0 & - & - & 4 & 9,1 & \\
\hline
\end{tabular}

* Significancia estadística considerando $p<0.05$

Tabla 2. Barreras utilizadas por los odontólogos en todos los Estados

\begin{tabular}{|c|c|c|c|c|c|c|}
\hline \multirow[b]{3}{*}{ Barreras } & \multicolumn{6}{|c|}{ Estados } \\
\hline & \multicolumn{2}{|c|}{$\mathrm{Si}$} & \multicolumn{2}{|c|}{ No } & \multicolumn{2}{|c|}{ NAP* } \\
\hline & n & $\%$ & $\mathbf{n}$ & $\%$ & $\mathbf{n}$ & $\%$ \\
\hline Gorro quirúrgico & 173 & 69,1 & 46 & 18,5 & 31 & 12,4 \\
\hline Gorro desechable & 174 & 69,6 & 45 & 18,0 & 31 & 12,4 \\
\hline Protección ocular & 216 & 86,3 & 3 & 1,3 & 31 & 12,4 \\
\hline Careta & 208 & 83,3 & 11 & 4,3 & 31 & 12,4 \\
\hline Mascarilla quirúrgica (triple capa) & 163 & 65,3 & 56 & 22,3 & 31 & 12,4 \\
\hline Mascarilla alta filtración KN95, N95 & 199 & 79,4 & 21 & 8,2 & 31 & 12,4 \\
\hline Respiradores & 31 & 12,5 & 188 & 75,1 & 31 & 12,4 \\
\hline Cubre calzado & 91 & 36,5 & 128 & 51,1 & 31 & 12,4 \\
\hline Guantes & 211 & 84,5 & 8 & 3,0 & 31 & 12,4 \\
\hline Doble guante & 98 & 39,1 & 121 & 48,5 & 31 & 12,4 \\
\hline Bata desechable & 170 & 67,8 & 49 & 19,7 & 31 & 12,4 \\
\hline Traje aislante & 128 & 51,1 & 91 & 36,5 & 31 & 12,4 \\
\hline Babero & 191 & 76,4 & 28 & 11,2 & 31 & 12,4 \\
\hline Campos desechables & 214 & 85,5 & 5 & 2,1 & 31 & 12,4 \\
\hline Plástico auto-adherente & 185 & 73,9 & 34 & 13,7 & 31 & 12,4 \\
\hline Funda desechable & 135 & 54,1 & 84 & 33,5 & 31 & 12,4 \\
\hline
\end{tabular}

*NAP $=$ No he atendido pacientes 
Tabla 3. Barreras utilizadas por los odontólogos de Zacatecas

\begin{tabular}{|c|c|c|c|c|c|c|}
\hline \multirow[b]{3}{*}{ Barreras } & \multicolumn{6}{|c|}{ Zacatecas } \\
\hline & \multicolumn{2}{|c|}{$\mathrm{Si}$} & \multicolumn{2}{|c|}{ No } & \multicolumn{2}{|c|}{ NAP* $^{*}$} \\
\hline & $\mathbf{n}$ & $\%$ & $\mathbf{n}$ & $\%$ & $\mathbf{n}$ & $\%$ \\
\hline Gorro quirúrgico & 34 & 68,0 & 10 & 20,0 & 6 & 12,0 \\
\hline Gorro desechable & 32 & 64,0 & 12 & 24,0 & 6 & 12,0 \\
\hline Protección ocular & 41 & 82,0 & 3 & 6,0 & 6 & 12,0 \\
\hline Careta & 42 & 84,0 & 2 & 4,0 & 6 & 12,0 \\
\hline Mascarilla quirúrgica (triple capa) & 35 & 70,0 & 9 & 18,0 & 6 & 12,0 \\
\hline Mascarilla alta filtración KN95, N95 & 39 & 78,0 & 5 & 10,0 & 6 & 12,0 \\
\hline Respiradores & 6 & 12,0 & 38 & 76,0 & 6 & 12,0 \\
\hline Cubre calzado & 15 & 30,0 & 29 & 58,0 & 6 & 12,0 \\
\hline Guantes & 42 & 84,0 & 2 & 4,0 & 6 & 12,0 \\
\hline Doble guante & 11 & 22,0 & 33 & 66,0 & 6 & 12,0 \\
\hline Bata desechable & 39 & 78,0 & 5 & 10,0 & 6 & 12,0 \\
\hline Traje aislante & 23 & 46,0 & 21 & 42,0 & 6 & 12,0 \\
\hline Babero & 41 & 82,0 & 3 & 6,0 & 6 & 12,0 \\
\hline Campos desechables & 42 & 84,0 & 2 & 4,0 & 6 & 12,0 \\
\hline Plástico auto-adherente & 33 & 66,0 & 11 & 220 & 6 & 12,0 \\
\hline Funda desechable & 22 & 44,0 & 22 & 44,0 & 6 & 12,0 \\
\hline
\end{tabular}

* NAP $=$ No he atendido pacientes

Tabla 4. Barreras utilizadas por los odontólogos de Sinaloa

\begin{tabular}{|c|c|c|c|c|c|c|}
\hline \multirow[b]{3}{*}{ Barreras } & \multicolumn{6}{|c|}{ Sinaloa } \\
\hline & \multicolumn{2}{|c|}{$\mathrm{Si}$} & \multicolumn{2}{|c|}{ No } & \multicolumn{2}{|c|}{ NAP* } \\
\hline & $\mathbf{n}$ & $\%$ & $\mathbf{n}$ & $\%$ & $\mathbf{n}$ & $\%$ \\
\hline Gorro quirúrgico & 35 & 70,0 & 10 & 20,0 & 5 & 10,0 \\
\hline Gorro desechable & 38 & 76,0 & 7 & 14,0 & 5 & 10,0 \\
\hline Protección ocular & 45 & 90,0 & - & - & 5 & 10,0 \\
\hline Careta & 43 & 86,0 & 2 & 4,0 & 5 & 10,0 \\
\hline Mascarilla quirúrgica (triple capa) & 33 & 66,0 & 12 & 24,0 & 5 & 10,0 \\
\hline Mascarilla alta filtración KN95, N95 & 44 & 88,0 & 1 & 2,0 & 5 & 10,0 \\
\hline Respiradores & 6 & 12,0 & 39 & 78,0 & 5 & 10,0 \\
\hline Cubre calzado & 20 & 40,0 & 25 & 50 & 5 & 10,0 \\
\hline Guantes & 45 & 90,0 & - & - & 5 & 10,0 \\
\hline Doble guante & 22 & 44,0 & 23 & 46,0 & 5 & 10,0 \\
\hline Bata desechable & 33 & 66,0 & 12 & 24,0 & 5 & 10,0 \\
\hline Traje aislante & 33 & 66,0 & 12 & 24,0 & 5 & 10,0 \\
\hline Babero & 33 & 66,0 & 12 & 24,0 & 5 & 10,0 \\
\hline Campos desechables & 44 & 88,0 & 1 & 2,0 & 5 & 10,0 \\
\hline Plástico auto-adherente & 36 & 72,0 & 9 & 18,0 & 5 & 10,0 \\
\hline Funda desechable & 28 & 56,0 & 17 & 34,0 & 5 & 10,0 \\
\hline
\end{tabular}

*NAP $=$ No he atendido pacientes 
Franco-Trejo et al.

Tabla 5. Barreras utilizadas por los odontólogos de Veracruz

\begin{tabular}{|c|c|c|c|c|c|c|}
\hline \multirow[b]{3}{*}{ Barreras } & \multicolumn{6}{|c|}{ Veracruz } \\
\hline & \multicolumn{2}{|c|}{$\mathrm{Si}$} & \multicolumn{2}{|c|}{ No } & \multicolumn{2}{|c|}{ NAP* } \\
\hline & n & $\%$ & $\mathbf{n}$ & $\%$ & n & $\%$ \\
\hline Gorro quirúrgico & 33 & 66,0 & 10 & 20,0 & 7 & 14,0 \\
\hline Gorro desechable & 35 & 70,0 & 8 & 16,0 & 7 & 14,0 \\
\hline Protección ocular & 43 & 86,0 & - & - & 7 & 14,0 \\
\hline Careta & 41 & 82,0 & 2 & 4,0 & 7 & 14,0 \\
\hline Mascarilla quirúrgica (triple capa) & 30 & 60,0 & 13 & 26,0 & 7 & 14,0 \\
\hline Mascarilla alta filtración KN95, N95 & 38 & 76,0 & 5 & 10,0 & 7 & 14,0 \\
\hline Respiradores & 10 & 20,0 & 33 & 66,0 & 7 & 14,0 \\
\hline Cubre calzado & 19 & 38,0 & 24 & 48,0 & 7 & 14,0 \\
\hline Guantes & 42 & 84,0 & 1 & 2,0 & 7 & 14,0 \\
\hline Doble guante & 17 & 34,0 & 26 & 52,0 & 7 & 14,0 \\
\hline Bata desechable & 36 & 72,0 & 7 & 14,0 & 7 & 14,0 \\
\hline Traje aislante & 19 & 38,0 & 24 & 48,0 & 7 & 14,0 \\
\hline Babero & 39 & 78,0 & 4 & 8,0 & 7 & 14,0 \\
\hline Campos desechables & 42 & 84,0 & 2 & 4,0 & 7 & 14,0 \\
\hline Plástico auto-adherente & 37 & 74,0 & 6 & 12,0 & 7 & 14,0 \\
\hline Funda desechable & 31 & 62,0 & 12 & 24,0 & 7 & 14,0 \\
\hline
\end{tabular}

*NAP $=$ No he atendido pacientes

Tabla 6. Barreras utilizadas por los odontólogos de Tlaxcala

\begin{tabular}{|c|c|c|c|c|c|c|}
\hline \multirow[b]{3}{*}{ Barreras } & \multicolumn{6}{|c|}{ Tlaxcala } \\
\hline & \multicolumn{2}{|c|}{$\mathrm{Si}$} & \multicolumn{2}{|c|}{ No } & \multicolumn{2}{|c|}{ NAP* } \\
\hline & $\mathrm{n}$ & $\%$ & $\mathbf{n}$ & $\%$ & n & $\%$ \\
\hline Gorro quirúrgico & 35 & 70,0 & 8 & 16,0 & 7 & 14,0 \\
\hline Gorro desechable & 32 & 64,0 & 11 & 22,0 & 7 & 14,0 \\
\hline Protección ocular & 43 & 86,0 & - & - & 7 & 14,0 \\
\hline Careta & 41 & 82,0 & 2 & 4,0 & 7 & 14,0 \\
\hline Mascarilla quirúrgica (triple capa) & 32 & 64,0 & 11 & 22,0 & 7 & 14,0 \\
\hline Mascarilla alta filtración KN95, N95 & 36 & 72,0 & 7 & 14,0 & 7 & 14,0 \\
\hline Respiradores & 4 & 8,0 & 39 & 78,0 & 7 & 14,0 \\
\hline Cubre calzado & 19 & 38,0 & 24 & 48,0 & 7 & 14,0 \\
\hline Guantes & 39 & 78,0 & 4 & 8,0 & 7 & 14,0 \\
\hline Doble guante & 27 & 54,0 & 16 & 32,0 & 7 & 14,0 \\
\hline Bata desechable & 32 & 64,0 & 11 & 22,0 & 7 & 14,0 \\
\hline Traje aislante & 26 & 52,0 & 17 & 34,0 & 7 & 14,0 \\
\hline Babero & 42 & 84,0 & 1 & 2,0 & 7 & 14,0 \\
\hline Campos desechables & 42 & 84,0 & 1 & 2,0 & 7 & 14,0 \\
\hline Plástico auto-adherente & 40 & 80,0 & 3 & 6,0 & 7 & 14,0 \\
\hline Funda desechable & 29 & 58,0 & 14 & 28,0 & 7 & 14,0 \\
\hline
\end{tabular}

*NAP $=$ No he atendido pacientes 
Tabla 7. Barreras utilizadas por los odontólogos de Puebla

\begin{tabular}{|c|c|c|c|c|c|c|}
\hline \multirow[b]{3}{*}{ Barreras } & \multicolumn{6}{|c|}{ Puebla } \\
\hline & \multicolumn{2}{|c|}{ Si } & \multicolumn{2}{|c|}{ No } & \multicolumn{2}{|c|}{$\mathrm{NAP}^{*}$} \\
\hline & $\mathbf{n}$ & $\%$ & $\mathbf{n}$ & $\%$ & $\mathbf{n}$ & $\%$ \\
\hline Gorro quirúrgico & 36 & 72,0 & 8 & 16,0 & 6 & 12,0 \\
\hline Gorro desechable & 38 & 76,0 & 6 & 12,0 & 6 & 12,0 \\
\hline Protección ocular & 44 & 88,0 & - & - & 6 & 12,0 \\
\hline Careta & 41 & 82,0 & 3 & 6,0 & 6 & 12,0 \\
\hline Mascarilla quirúrgica (triple capa) & 33 & 66,0 & 11 & 22,0 & 6 & 12,0 \\
\hline Mascarilla alta filtración KN95, N95 & 42 & 84,0 & 2 & 4,0 & 6 & 12,0 \\
\hline Respiradores & 5 & 10,0 & 39 & 78,0 & 6 & 12,0 \\
\hline Cubre calzado & 18 & 36,0 & 26 & 52,0 & 6 & 12,0 \\
\hline Guantes & 44 & 88,0 & - & - & 6 & 12,0 \\
\hline Doble guante & 21 & 42,0 & 23 & 46,0 & 6 & 12,0 \\
\hline Bata desechable & 27 & 54,0 & 17 & 34,0 & 6 & 12,0 \\
\hline Traje aislante & 27 & 54,0 & 17 & 34,0 & 6 & 12,0 \\
\hline Babero & 35 & 70,0 & 9 & 18,0 & 6 & 12,0 \\
\hline Campos desechables & 44 & 88,0 & - & - & 6 & 12,0 \\
\hline Plástico auto-adherente & 39 & 78,0 & 5 & 10,0 & 6 & 12,0 \\
\hline Funda desechable & 24 & 48,0 & 20 & 40,0 & 6 & 12,0 \\
\hline
\end{tabular}

*NAP $=$ No he atendido pacientes

Zacatecas y Sinaloa respondieron que no las usan. Las respuestas obtenidas respecto al cambio de funda, plástico adherente y babero fue que el 90,6\% (226) con cada paciente, $4,7 \%$ (12) al término de la jornada y $0,9 \%$ (2) cuando se dañan y 3,9\% (19) no utilizan. En los estados se encontraron valores mayores a $90 \%$, en cuatro de ellos con el cambio con cada paciente, siendo Puebla el que respondió con el valor más bajo con $78,8 \%$; quien no lo utiliza estuvo presente en todos los estados siendo Puebla el más elevado con un $9,1 \%$ continuando con Veracruz en $6,0 \%$.

Se preguntó sobre la frecuencia del cambio de gorro, careta, mascarilla y bata, respondiendo que con cada paciente Tlaxcala con 58\% (29), Sinaloa y Zacatecas 50\% (25) mientras Veracruz y Puebla con $46.0 \%$ (23) y $24 \%$ (17), respectivamente. En la opción al término de la jornada fue de 64\% (32) Puebla, 54\% (27) Veracruz, 48\% (24) Sinaloa, 46\% (23) Zacatecas, 40\% (20) Tlaxcala. Cuando se dańan o no se utilizan, una persona respondió afirmativa en cada estado, a excepción de Veracruz. Además, se preguntó si se incrementó el uso de barreras durante la pandemia, a lo que respondieron que sí en Tlaxcala con un $96 \%$ (48), Sinaloa 94\% (47), Zacatecas 90\% (45), Puebla y Veracruz 88\% (44), sólo cinco personas señalaron que no las aumentaron y el resto no han atendido pacientes.

\section{Discusión}

Dentro de la normatividad de México para dicha área, se tiene la NOM-013-SSA2-2015, para la prevención y control de enfermedades bucales ${ }^{20}$, la guía para la atención estomátológica en personas con VIH ${ }^{21}$ (SS, 2015) y el Manual para la Prevención y control de infecciones y riesgos profesionales en la práctica estomatológica en la República Mexicana ${ }^{22}$ (SS, 2003); mismas que los profesionales de esta área deben estar al día para el apego a los criterios establecidos. A mediados del 2020 se publicó por parte de la subdirección de prevención y protección la semaforización para retomar la práctica odontológica ${ }^{18,19}$; sin embargo, aún existe falta de conciencia y apego a lo establecido en dichos documentos, ya que las cifras encontradas en los resultados en el total de los participantes indican que las principales barreras recomendadas como lentes, mascarilla, guantes y bata oscilan de $65 \%$ a $86 \%$, similar a lo encontrado por Díaz ${ }^{7}$ y Serrano et al. ${ }^{10}$

Como ya se mencionó, los profesionales de la odontología tienen mayor riesgo de contagio para las enfermedades transmisibles, desde hace años se está haciendo énfasis en el uso de barreras de protección como una medida de prevención y/o control. Peng et al. ${ }^{5}$ señaló que el uso adecuado de gafas protectoras, mascarillas, guantes, gorros, protectores faciales y ropa protectora, durante todo el tratamiento odontológico, solo reducirán el riesgo de transmisión y no pueden proporcionar una barrera a prueba de ellas. Al comparar con lo publicado por Camargo et al. ${ }^{6}$ y Barbosa et al. ${ }^{13}$, el uso de bata fue menor en los mexicanos en un $67,8 \%$ a diferencia del $91,35 \%$ y $100 \%$, respectivamente; siendo 
más prevalente en Zacatecas con 78\%. En México el uso de gorro fue de $69,1 \%$ el desechable y $69,6 \%$ quirúrgico menor que Camargo et al. ${ }^{6}$, el desechable fue más utilizado en Sinaloa, Veracruz y Puebla. Respecto a en uso de la mascarilla fue mayor a lo publicado por Camargo et al. ${ }^{6}$, pero menor a lo que encontró Barbosa et al. ${ }^{13}$ en cuanto a la N95, pero mayor para la quirúrgica. La comparación entre los estados señaló que existe mayor uso de la mascarilla de alta filtración en todos los odontológos. El uso de guantes con cada paciente fue menor México que lo de Camargo et al. ${ }^{6}$, Sinaloa obtuvo el porcentaje más elevado con $90 \%$ y el doble guante con $44,0 \%$ en la misma entidad.

Según los datos encontrados se concluyó que el uso de las barreras de protección fue elevado; sin embargo, se espera que se incremente la utilización de las mismas para la prevención de las enfermedades infecciosas en especial por SARS-CoV-2. Se identificaron diferencias entre los estados respecto a la edad, estado civil y el nivel de estudios. El equipo más empleado fue la protección ocular, careta, mascarillas, guantes, bata y campos desechables; dichos equipos son cambiados posterior a la atención de cada paciente o al término de la jornada laboral, sin embargo, es necesario de incremente el que sea después de la atención individual.

\section{Referencias bibliográficas}

1. Alvarado E. Cumplimiento de las normas de bioseguridad en enfermedades transmisibles en la práctica clínica Odontológica por Cirujanos Dentistas miembros de Sociedades Científicas Odontológicas del Perú en el 2015 [tesis de magister]. [Perú]: Escuela de Posgrado, Universidad César Vallejo. [internet]. 2016 [citado $2021 \mathrm{feb}$ 08] 103 p. Disponible en: http://repositorio.ucv.edu.pe/ bitstream/handle/20.500.12692/7314/Mu\%c3\%blos_ ERA.pdf?sequence $=1$ \&isAllowed $=\mathrm{y}$

2. Pareja G. Riesgo de transmisión de enfermedades infecciosas en la clínica dental. RCOE [Internet]. 2004 [citado 2021 Dic 03]; 9(3):313-321. Disponible en: http://scielo.isciii.es/scielo.php?script=sci_arttext\&pi$\mathrm{d}=$ S1138-123X2004000300005\&lng=es.

3. World Health Organization. Middle East respiratory syndrome coronavirus (MERS-CoV): summary of current situation, literature update and risk assessment. World Health Organization. [internet]. 2015 july [citado 2021 mar 03]. 7p. Disponible en: https://apps.who.int/ iris/bitstream/handle/10665/179184/WHO_MERS_ RA_15.1_eng.pdf?sequence $=2$ \&isAllowed $=\mathrm{y}$

4. Kampf G, Todt D, Pfaender S, Steinmann E. Persistence of coronaviruses on inanimate surfaces and their inactivation with biocidal agents. Journal of Hospital Infection, [internet]. 2020 [citado $2021 \mathrm{feb}$ 18]. 104(3):246-51. Disponible en: https://www. journalofhospitalinfection.com/action/showPdf?pi$\mathrm{i}=$ S0195-6701\%2820\%2930046-3 Doi: $10.1016 / \mathrm{j}$. jhin.2020.01.022

5. Peng X, Xu X, Li Y, Cheng L, Zhou X, Ren B. Transmission routes of 2019-nCoV and controls in dental practice. International Journal of Oral Science, [internet]. 2020 [citado 2021 mar 03]. 12(9). Disponible en: https://www.nature.com/articles/s41368-020-0075-9 DOI: $10.1038 / s 41368-020-0075-9$

6. Camargo J, Sierra Y, Sierra MC. Uso de implementos y medidas de bioseguridad en las clinicas odontologicas de Bucaramanga de la Universidad Santo Tomás en el segundo semestre del ańo 2015. [tesis de licenciatura]. [Colombia]. Facultad de odontología, Universidad Santo Tomás. [internet]. 2016 [citado $2021 \mathrm{feb} 08$ ], 55p. Disponible en: https://repository.usta.edu.co/bitstream/ handle/11634/1790/2016-CamargoJenny\%20Viviana-VeraYesenia-SierraMariaCamila-trabajodegrado.pdf? sequence $=1$ \&isAllowed $=y$

7. Díaz V. Grado de aplicación de precauciones estándar durante la atención de pacientes por estudiantes de programa de especialización profesional en endodoncia. [tesis de licenciatura]. [Chile]. Facultad de Odontología, Universidad de Chile. [internet]. 2016 [citado 2021 feb 18] 98p. Disponible en: http://repositorio.uchile. cl/bitstream/handle/2250/141904/Grado-de-aplicaci\%c3\%b3n-de-precauciones-est $\%$ c3\%alndar-durante-la-atenci\%c3\%b3n-de-pacientes.pdf?sequence $=1 \&$ isAllowed $=\mathrm{y}$

8. Paz M. Conocimientos, actitudes y prácticas de normas de bioseguridad y riesgo biológico en odontólogos de práctica privada de tres ciudades de Nicaragua. Odontología Sanmarquina [internet]. 2019 [citado 2021 may 20] 22(1):19-25. Disponible en: https://revistasinvestigacion.unmsm.edu.pe/index.php/odont/article/ view/15839 DOI:10.15381/os.v22i1.15859

9. Assiri KI, Naheeda, Kaleem S, Ibrahim M, Alam T, Asif $S$. Knowledge, attitude, and practice of infection control among dental students in King Khalid University, Abha. J Int Oral Health [internet]. $2018 \mathrm{Apr}$ [citado $2021 \mathrm{mar}$ 12] 10:83-7 Disponible en: https://www.jioh.org/article. asp? issn $=0976-7428$; year $=2018$; volume $=10$;issue $=2 ;$ spage $=83$; epage $=87$; aulast $=$ Assiri \#google_vignette

10. Serrano S, Margot S. Normas de bioseguridad por COVID-19 y satisfacción del usuario en consultorios odontológicos privados en la ciudad de Abancay, Apurímac, 2020. [tesis de maestría]. [Perú]: Escuela de Posgrado, Universidad César Vallejo. [internet]. 2021 [citado 2021 may 20] 65p. Disponible en: https://repositorio.ucv.edu. pe/bitstream/handle/20.500.12692/57742/Soria_SSMSD.pdf?sequence $=4$

11. Pastor T. Nivel de conocimiento en cirujanos dentistas sobre el uso de EPPS de bioseguridad en la atención odontológica, Cajamarca -2020. [tesis de licencitura]. [Perú]: Escuela Profesional de Estomatología, Universidad Privada Antenor Orrego. [internet]. 2021 [citado 2021 may 20]. 43p. Disponible en: https://repositorio. upao.edu.pe/bitstream/20.500.12759/7406/1/REP_ THALIA.PASTOR_CONOCIMIENTO.EN.CIRUJANOS.DENTISTAS.pdf

12. Vazquez S, Rodríguez M, Monroy R, Cardenas R. Development and validation of an instrument to assess adherence to occupational health protocols in dentistry. J Dent Educ. [internet]. 2021 [citado 2021 jun 03] 331340. Disponible en: https://onlinelibrary.wiley.com/doi/ epdf/10.1002/jdd.12454 DOI:10.1002/jdd.12456

13. Barbosa L, Agudelo A, Atuesta M, Ariza J, Plaza S. Modificación de la práctica odontológica, seguimiento a protocolos y percepción de riesgo de los odontólogos 
durante la pandemia de COVID-19 en Colombia: estudio de corte transversal. Rev Fac Odontol Univ Antioq. [internet]. 2021 [citado 2021 jun 05] 33(1). Disponible en: https://revistas.udea.edu.co/index.php/odont/article/view/343942/20804591 DOI: 10.17533/udea.rfo. v33n1a2

14. Diario Oficial de la Federación. ACUERDO número 02/03/20 por el que se suspenden las clases en las escuelas de educación preescolar, primaria, secundaria, normal y demás para la formación de maestros de educación básica del Sistema Educativo Nacional, así como aquellas de los tipos medio superior y superior dependientes de la Secretaría de Educación Pública. DOF-SEGOB. [internet]. 2020 mar [citado 2021 sep 29]. Disponible en: https:// www.dof.gob.mx/nota_detalle.php?codigo $=5589479 \&-$ fecha $=16 / 03 / 2020$

15. Cázares L, Peraldi M, Aneyba L, Soto D. Impacto económico en el medio odontológico durante la pandemia del COVID-19: revisión integradora. Revista ADM. [internet]. 2021 [citado 2021 sep 29] 78(1):42-47. Disponible en: https://www.medigraphic.com/pdfs/adm/ od-2021/od211g.pdf DOI:10.35366/98386.

16. Secretaria de Relaciones Exteriores. Datos Básicos de México. [internet]. 2016 [citado 2021 dic 03]. Disponible en: https://embamex.sre.gob.mx/nigeria/index.php/ es/mexico-informacion

17. Diario Oficial de la Federación. Reglamento de la Ley General de salud en materia de investigación para la salud. [internet]. 2014 [citado 2021 feb 02]. Disponible en: http://www.conbioetica-mexico.salud.gob.mx/descargas/pdf/normatividad/normatinacional/10._NAL. Reglamento_de_Investigacion.pdf
18. Secretaria de Salud. Semaforo de recomendaciones para la atención de la práctica odontológica. [internet]. 2020 [citado 2021 abr 15]. Disponible en: https://www.odontologos.mx/descargas/sesiones/2020/secretaria-salud/ SEMAFORO-DE-ATENCION-PARA-LA-PRACTICA-ODONTOLOGICA-general-2020.pdf

19. Secretaria de Salud.Recomendaciones para la práctica odontológica. [internet]. 2020 [citado $2021 \mathrm{abr} 15$ ]. Disponible en: https://www.odontologos.mx/descargas/ sesiones/2020/04-abril/recomendaciones-odontologicas-COVID-2020.pdf

20. Secretaria de Salud. Norma oficial mexicana NOM013-SSA2-2015, para la prevención y control de enfermedades bucales. [internet]. 2016 [citado 2021 feb 08]. Disponible en: http://www.cenaprece.salud.gob.mx/programas/interior/saludbucal/descargas/pdf/NORMA013. pdf

21. Secretaria de Salud. Guía para la atención estomatológica en personas con VIH. [internet]. 2013 [citado 2021 feb 08]. Disponible en: http://www.cenaprece.salud. gob.mx/programas/interior/saludbucal/descargas/pdf/ GUIA_ESTOMATOLOGICA2015.pdf

22. Secretaria de Salud. Manual para la prevención y control de infecciones y riesgos profesionales en la práctica estomatológica en la República Mexicana. [internet]. 2003 [citado 2021 feb 08]. Disponible en: https://salud. edomex.gob.mx/isem/documentos/temas_programas/ sbucal/Manuales/Manual-Prev.-y-Control-de-Infecciones-profesionales $\% 5 \mathrm{~b} 1 \% 5 \mathrm{~d}$.pdf 